\title{
Prostatakarzinompatienten wünschen sich regelmäßige Kontrollen - auch bei langem Therapieintervall
}

— Im Fokus einer Umfrage der Otto-vonGuericke-Universität Magdeburg unter Prostatakarzinompatienten steht die Rolle des Arztes bei der Patientenbetreuung. Befragt wurden 75 Patienten mit Prostatakarzinom unter antihormoneller Therapie aus 19 urologischen Arztpraxen zum Thema "Lebenszufriedenheit“. Die Befragung ergab, dass über $60 \%$ der Befragten ein langes Therapieintervall bevorzugen. Die Mehrheit wünscht sich eine einfach anwendbare Behandlung, die sie in ihrem Alltag so wenig wie möglich beeinträchtigt. $86 \%$ der Befragten sprachen sich für eine vierteljährliche Betreuung mit Kontrolluntersuchungen und Gesprächen beim Arzt aus. Für die meisten Betroffenen ist dabei in erster Linie die Zusi- cherung des Arztes wichtig, dass mit ihrem Gesundheitszustand „alles in Ordnung“ sei. Viele Patienten möchten sich also nicht ständig mit ihrer Krankheit auseinandersetzen, jedoch während der Therapie regelmäßig über ihren Gesundheitszustand informiert werden. Diesen Bedürfnissen wird in der Hormon-Suppressionstherapie das Jahresimplantat Histrelin $\left(\right.$ Vantas $^{\circledR}$ ) gerecht. Es wird als Langzeittherapie bei Patienten im fortgeschrittenen Stadium der Erkrankung eingesetzt. Histrelin gibt zwölf Monate lang kontinuierlich seinen Wirkstoff frei und sorgt für einen konstant niedrigen Testosteronspiegel ohne Miniflares. Nach einem Jahr kann das Implantat entweder entfernt oder bei Bedarf umgehend durch ein neues ersetzt werden.
Um Ärzte und Patienten durch ein Therapiejahr zu begleiten und die Arzt-Patientenbindung zu unterstützen, hat Orion Pharma das Vantas-Jahresprogramm ${ }^{\circledast}$ entwickelt. Das Servicepaket enthält unter anderem sieben Informationsbroschüren, die neben Informationen über Erkrankung und Therapiemöglichkeiten auch Wissenswertes rund um das Leben mit Krebs bieten. Weitere Informationen zu Vantas ${ }^{\circledast}$ und dem Jahresprogramm finden Ärzte unter www.vantas-pca.de. Patienten steht unter www.jahresprogrammprostatakrebs.de ein Informationsportal zur Verfügung.

Nach Informationen von

Orion Pharma, Hamburg

\section{Gesunde Hände, korrekte Desinfektion}

— Nur eine intakte Haut lässt sich sicher desinfizieren, daher trägt ein konsequenter Hautschutz zur Infektionsprävention bei. Bereits kleinste Hautrisse und Mikrotraumen führen zu Hautbrennen bei der Händedesinfektion und erschweren ihre korrekte
Durchführung. Davon abgesehen sind Hautschäden auch eine potenzielle Nische für Erreger - beides erhöht das Risiko nosokomialer Infektionen. Gerade im Gesundheitswesen gehören Erkrankungen der Haut zu den häufigsten Berufserkrankungen.

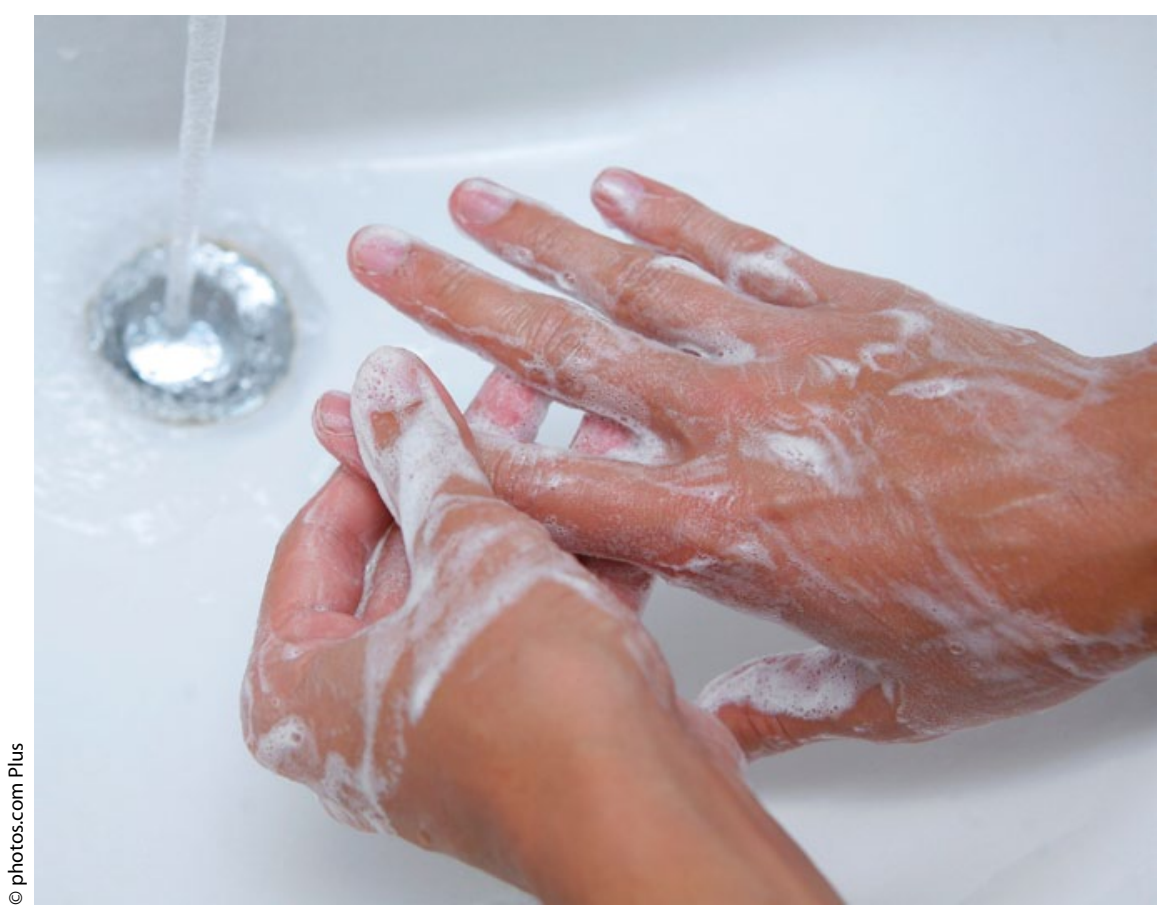

Nur wenn die Haut gesund ist klappt die Handdesinfektion richtig.
Vor diesem Hintergrund hat die Paul Hartmann AG die Kampagne "Gesunde Hände schützen" ins Leben gerufen und macht damit gezielt auf diesen Zusammenhang aufmerksam. Die Informationskampagne unterstützt medizinische Einrichtungen dabei, den Haut- und Infektionsschutz zu erhöhen. Zahlreiche Aktionen darunter auch eine Website zum Thema sind noch in Planung.

Praktische Lösungen bietet das Unternehmen auch mit seinem HändehygieneSystem: die Hände-Desinfektionsmittel der Sterilium ${ }^{\oplus}$-Range, die Untersuchungs- und Schutzhandschuhe der Peha-soft ${ }^{\circledR}$-Range sowie die Hände-Pflege- und HändeReinigungsprodukte der Baktolan ${ }^{\circledast}$ - und Baktolin ${ }^{\oplus}$-Serie. Alle Produkte sind hautverträglich und wurden wissenschaftlich begutachtet. Dabei wurde unter anderem darauf geachtet, ob die einzelnen Komponenten miteinander kompatibel sind, denn Studien belegen, dass Hände-Pflegeprodukte beispielsweise die Effizienz von Hände-Desinfektionsmitteln beeinträchtigen können. Die Kompatibilitätsgutachten stehen also für ein hohes Maß an Anwendersicherheit.

Nach Informationen von

Paul Hartmann, Heidenheim 